\title{
La alzheimerización de la vejez. Aportes de una etnografía de los cuidados
}

\author{
VIOLETTA CESANELLI Y SUSANA MARGULIES
}

En las sociedades occidentales modernas, envejecer se asocia a degeneración y a la cercanía de la muerte. En muchos países, las enfermedades degenerativas se consideran un problema grave de salud pública y las personas que las padecen constituyen un peso para la familia y la sociedad. En la antropología analizamos cómo la vejez dejó de ser una etapa de la vida para transformarse en un problema médico por medio de la construcción de la categoría diagnóstica de "enfermedad de Alzheimer". Presentamos resultados parciales de una investigación etnográfica efectuada entre 2011 y 2013, en Francia e Italia, con seis mujeres diagnosticadas con Alzheimer, con el propósito de explorar la experiencia de vivir con esta enfermedad y la importancia de los cuidados.

PALABRAS CLAVE: biomedicina, conductas de cuidado, criterios de diagnóstico, Alzheimer, antropología de la salud

\section{The Alzheimerization of Aging. Contributions of the Ethnography of Care}

In Western modern societies ageing is associated with degeneration and proximity of death. In many countries in Europe and in the United States, degenerative diseases are considered a serious public health problem and people who suffer them represent a burden for their family and for the society. Applying an anthropological perspective, we analyze how "being old" become a medical and social problem through the construction of "Alzheim-

Violetta CesanelLI Universidad de Buenos Aires, Buenos Aires, Argentina cesanelli.violetta@hotmail.fr

SusANA MARgulies Universidad de Buenos Aires, Buenos Aires, Argentina sumargulies@gmail.com er's disease" as a diagnostic category. We close with partial results of an ethnographic investigation from 2011 to 2013 in France and Italy, about the experience of six women living with Alzheimer's disease and the importance of being cared.

KEYWORDS: biomedicine, caring behavior, diagnostic criteria, Alzheimer, anthropology of health 


\section{Introducción}

\section{A}

partir de la segunda mitad del siglo XX, las sociedades occidentales se transformaron de manera radical debido a un doble movimiento demográfico: el descenso de la fecundidad y la mortalidad. Este fenómeno tuvo como consecuencia el envejecimiento de la población, que contribuyó a modificar la percepción social de la vejez y la muerte. A principios de esa centuria la muerte no tenía edad, pues las malas condiciones de vida y las guerras frecuentes hacían que la muerte sobreviniera en cualquier momento, pero durante los dos últimos siglos la esperanza de vida de los individuos creció de manera ininterrumpida en gran parte del mundo. En Francia, por ejemplo, la duración media de vida de un hombre pasó de 38.8 años, en 1820 (Peatrik, 2003: 167), a 80 años, en 2015 (Tavernier, 2016: 34). Gracias al mejoramiento de las condiciones generales de vida de las poblaciones, el establecimiento de políticas de salud pública y el perfeccionamiento de las prácticas hospitalarias, el panorama sanitario de los países desarrollados cambió y las enfermedades infecciosas y cardiovasculares, que hasta ese momento constituían las causas principales de muerte, dejaron de ser fatales. En palabras que Anne-Marie Peatrik toma de Jacques Dupâquier, enfermar por estas causas dejó de ser sinónimo de muerte (2003: 167).

No morir joven significa también la posibilidad de envejecer. Por un lado, "la prolongación de la esperanza de vida devino un símbolo de modernidad y progreso" (Lafontaine, 2010: 6). Por el otro, implicó un aumento en la probabilidad de padecer una enfermedad degenerativa, como cáncer, Parkinson o Alzheimer, que, en el imaginario colectivo, llevan a la muerte después de un proceso por lo general largo, lento y doloroso. Así, como plantea Céline Lafontaine, la vejez comenzó a asociarse de manera directa a la degeneración y proximidad de la muerte, "se transformó en una plaga que hay que combatir" (2010: 7).

En este artículo efectuamos, en primer lugar, un análisis bibliográfico de la construcción de la categoría diagnóstica de "enfermedad de Alzheimer" y de 
cómo la vejez dejó de ser una etapa normal de la vida para transformarse en un problema médico. Discutimos el papel de la medicina, en específico de la psiquiatría y la gerontología, en el proceso de medicalización de la vejez, y planteamos algunas de las condiciones sociales y culturales que contribuyeron a hacer de la vejez una enfermedad mortal (Lafontaine, 2010). En la segunda parte presentamos resultados de la investigación etnográfica de Violetta Cesanelli para su tesis de maestría, llevada a cabo en 2013, en Francia e Italia, orientada a explorar la experiencia de vivir con la enfermedad de Alzheimer. Esa investigación incluyó el acompañamiento de seis mujeres que atravesaban fases de la enfermedad. En este artículo desarrollamos los casos de Mina, madame Gris y Constantina.

\section{Discusión}

\section{LA CONSTRUCCIÓN DE LA ENFERMEDAD}

El 26 de noviembre 1901, una mujer de 51 años de edad llamada Auguste D. se interna en un hospital de Frankfurt, especializado en enfermedades mentales y epilépticas. El doctor Aloïs Alzheimer le diagnostica una demencia acompañada por un trastorno de la orientación, alucinaciones, confusión mental y discapacidad psicosocial. Cinco años después, el 8 abril de 1906, Auguste D. muere. El doctor Alzheimer, quien se había mudado a Munich con su colega y amigo Emil Kraepelin, solicita a la dirección del hospital que le envíe el cerebro de la paciente para realizar una autopsia. Al abrirlo, el doctor constata que la anatomía del cerebro de su paciente estaba alterada. Lo describe como un órgano atrofiado que presenta signos de modificaciones ateroescleróticas y de degeneración neurofibrilar.

El mismo año, Aloïs Alzheimer revela por primera vez sus observaciones en la Conferencia número 37 de Psiquiatras del Suroeste de Alemania. En esa ocasión, describe el caso de Auguste D. como un nuevo tipo de demencia que genera una degradación progresiva del tejido cerebral y produce la pérdida de las facultades cognitivas de los pacientes. Luego, en un artículo publicado en 1907, el doctor Alzheimer es más preciso y habla de una "afección rara del córtex cerebral que aflige a las personas de menos de 65 años y la define como demencia presenil" (Ngatcha-Ribert, 2012: 48).

A pesar de su importancia, este descubrimiento no tiene impacto inmediato en la comunidad médica y científica, dado que las técnicas de conservación de los cerebros y los procedimientos para efectuar los exámenes microscópicos de histología para la identificación de patologías nerviosas que utilizaba el doctor Alzheimer se difundían con lentitud. Sin embargo, el libro que Kraepelin publica en 1903 suscita mayor interés entre los médicos. En él comenta el descubrimiento de su colega y reconoce lo que denomina la "enfermedad de Alzheimer", caracterizada por su fase presenil. A partir de ese momento, empiezan a aparecer en la literatura científica descripciones de la enfermedad. Sin embargo, los nuevos casos, 11 en cinco años, resultan problemáticos en términos nosológicos respecto de la definición inicial, pues algunos pacientes tenían más de 65 años de edad. Con esta variación nosológica, la enfermedad de Alzheimer pasa a ocupar un lugar importante en la literatura sobre demencia. A continuación, veremos cómo las modificaciones de la categoría diagnóstica de enfermedad de Alzheimer se asocian al proceso de medicalización de la vejez.

Entre las décadas de 1930 y 1950, los psiquiatras - primero estadounidenses; alemanes y franceses después - redefinen las relaciones entre neuropatologías y comportamientos al incorporar un nuevo grupo de pacientes hasta entonces víctimas de lo que se había denominado nihilismo terapéutico: las personas mayores (Ngatcha-Ribert, 2012: 111). En efecto, hasta la década de 1940, los psiquiatras estiman inapropiado tratar a una población de personas 


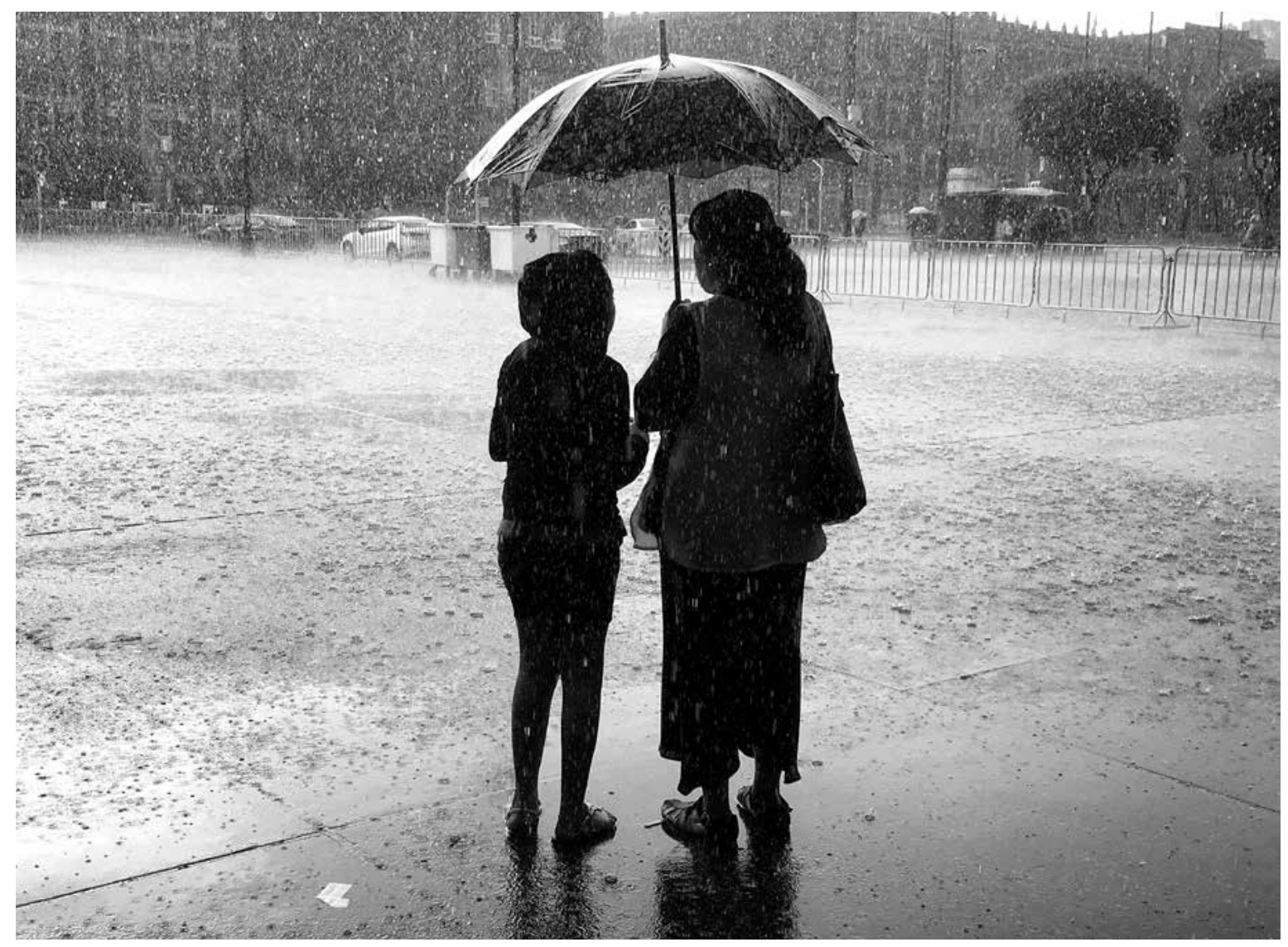

Prometeo Lucero • En medio del Zócalo, una familia se cubre sin éxito de la lluvia, abril de 2018.

mayores. A partir de entonces, la enfermedad de Alzheimer y la demencia, llamada senil en las personas mayores, empiezan a considerarse "enfermedades mentales inducidas por un proceso psico-dinámico que se desarrolla entre los individuos que envejecen y la sociedad" (Ballenger, 2003: 83). Según Jesse Ballenger, el objetivo de esta nueva perspectiva era dejar de calificar la enfermedad de Alzheimer y la demencia senil como síndromes para definirlas como "patologías específicas e identificables" (2003: 84). Por otro lado, contribuyó a que se abandonara la idea del trastorno cognitivo biológicamente inducido y a establecer el diagnóstico a partir de la identificación de los factores de vulnerabilidad psicosociales asociados a la biografía del sujeto y su personalidad.
Este proceso de patologización tuvo dos efectos importantes: el desarrollo de investigaciones y experimentaciones terapéuticas - terapias electroconvulsivas, medicamentos, terapias interactivas y de grupo, hormonas, vitaminas, terapias nutricionales, etc.—, y la cooperación entre psiquiatría y gerontología. De hecho, en ese momento se profesionalizó la primera generación de gerontólogos, con el propósito de "hacerse cargo de la vejez en la sociedad y de luchar contra la senilidad" (NgatchaRibert, 2012: 112).

A finales de la década de 1960, las perspectivas cambian de nuevo: lo biológico retoma importancia con lo genético en la explicación de estas patologías y desplaza los factores psicosociales. Según 
Katherine Bick (1994), la era moderna de la investigación sobre la enfermedad de Alzheimer empieza en 1963, con las primeras observaciones de los cerebros de pacientes diagnosticados con Alzheimer realizadas en Inglaterra y Estados Unidos con el microscopio electrónico, recién inventado. Estos avances permiten establecer que los cerebros de las personas enfermas de Alzheimer de menos de 65 años de edad y de quienes sufren de demencia senil presentan lesiones y características ultraestructurales muy parecidas (Katzman, 1976: 217-218). A partir de ese momento, las dos patologías se superponen cada vez más. La demencia senil, característica de las personas mayores de 65 años, es absorbida por la categoría diagnóstica Alzheimer y se transforma en “demencia senil de tipo Alzheimer". Más tarde se categoriza como "enfermedad de Alzheimer y enfermedades asociadas". Esta última denominación extiende la categoría diagnóstica a pacientes de 50 a 85 años de edad y borra la distinción entre presenilidad y senilidad. Conceptualmente, esto equivale a separar vejez de senilidad. En otras palabras, se define la senilidad como patológica, asociada a disfunciones cognitivas o comportamentales que antes se relacionaban con el envejecimiento normal. Richard Adelman (1995) se refiere a este fenómeno con el concepto de alzheimerization of aging, alzheimerización del envejecimiento. Así, dos figuras de la vejez se oponen: una considerada normal, que no incluye los síntomas de tipo Alzheimer, y la otra, patológica.

Si bien la alzheimerización de la vejez es un proceso que ocurre en el ámbito médico, también ha de analizarse en su amplitud como proceso social. $\mathrm{Al}$ alzheimerizar la vejez, la biomedicina introdujo una serie de normas y valores morales socialmente compartidos. Una de las múltiples funciones de la biomedicina moderna en la historia ha sido determinar las condiciones y modalidades de la vida social que son patológicas o anormales. La caracterización de prácticas y representaciones sociales en términos biomédicos redefine ciertos aspectos de la vida y los transforma en problemas médicos. Peter Conrad y Arthur Schneider llaman a este proceso "la medicalización de los fenómenos sociales" (1992: 328), por ejemplo, el parto, la menopausia, la obesidad, la angustia, la timidez, el consumo de alcohol, la hiperactividad en niños y la vejez, entre otros.

Como resultado de la medicalización de la vida en las sociedades occidentales, la biomedicina y las tecnologías médicas pasan a ocupar un lugar significativo en la vida cotidiana de los individuos y contribuyen a modelar la percepción social sobre la vejez.

\section{Alzheimer, una cuestión médica, social y política}

En muchos países de Europa y en Estados Unidos, la enfermedad de Alzheimer se considera un problema grave de salud pública, que se traduce en una población anciana y dependiente, un peso para la familia y la sociedad, que requiere medidas de atención drásticas. En este marco, la enfermedad se transforma en la representación de la demencia y la vejez patológica. Si bien esto es resultado de un cambio de categorías diagnósticas inducido por los médicos, en particular los psiquiatras y gerontólogos, Ballenger (2003) plantea que esta nueva concepción de la vejez se inscribe también en un proceso de transformaciones socioculturales, sin las cuales la enfermedad de Alzheimer no hubiera podido surgir como problema social, médico y político mayor.

Las sociedades occidentales modernas, denominadas civilizaciones tecnológicas por Jean-Philippe Pierron (2010: 101), se inscriben en un "cuadro de interpretación del cuerpo vivido" que valora la autonomía y exalta la productividad, la movilidad y la plasticidad del cuerpo y la mente. Guillaume Le Blanc explica que el proceso de medicalización y esta visión del cuerpo vivido trasforman las concepciones de la enfermedad, la muerte y la vejez al erigir como 
norma y símbolo de la modernidad "la salud perfecta" y "un cuerpo que puede ser todo el tiempo mejorado y siempre más productivo" (2002: 72).

Pero la salud perfecta no sólo implica un cuerpo que funciona, sino también una cognición perfecta. Stephen Post, médico estadounidense y profesor de ética médica en la Stony Brook University en Nueva York, sostiene en este sentido que las sociedades industriales modernas, que describe como hipercognitivas, conceden un peso cultural creciente al cerebro (2000: 155).

En la actualidad, la función cerebral tiene un papel clave en la distinción entre lo vivo y lo muerto. La determinación científica de la muerte tiene una larga trayectoria. Desde los croque-mort - los enterradores, traducido de manera literal como "mordedor de muerte"-, que en la Francia del siglo XVIII mordían al presunto muerto para asegurarse de su estado, hasta hoy, la muerte ha requerido determinaciones tecnológicas cada vez más precisas. En la actualidad, la muerte cerebral — brain-death - se considera el final de la vida (Lock, 2004: 136), pero esta definición es debatible y ambigua en algunas circunstancias, por ejemplo, en el caso del trasplante de órganos, cuando el cuerpo tiene que mantenerse con vida de manera mecánica para preservar los órganos (Lock, 2004). Así, se crean híbridos, living cadavers en la formulación de Lock: seres no vivos ni muertos por completo.

También la función cerebral tiene un papel central en la determinación del comienzo de la vida. En julio de 1982, The Warnock Committee (1984) se estableció en la cuidad de Londres con el propósito de examinar las investigaciones sobre fecundación y embriología humanas, atender las implicaciones sociales, éticas y legales de estos desarrollos, y hacer recomendaciones, pioneras de la regulación legislativa sobre la investigación embrionaria. El comité determinó un límite legal de 14 días después de la fecundación, adoptado después por otros países, durante los cuales el "pre-embrión” humano, ${ }^{1}$ en otras palabras, el producto inicial de la concepción, no se considera un organismo individual y puede estar in vitro o ser objeto de experimentación.

¿Por qué elegir como umbral el día 14? Al igual que con la precisión de la muerte, el funcionamiento del sistema nervioso central, en este caso su formación, determina la vida. Así, al día 14 se observa “el principio de la 'línea primitiva' o trazo de la espina vertebral [...]. Basándose en este 'hecho', la Human Fertilization and Embryology Authority de Gran Bretaña aprobó la experimentación científica con embriones" (Roca, 2013: 5).

La actividad cerebral y del sistema nervioso central pasan a ocupar un lugar de suma importancia en la determinación de la vida y la muerte biológicas. A partir del señalamiento de esa frontera, la sociedad y sus instituciones políticas y científicas toman decisiones que incluyen o excluyen individuos de la vida social.

\section{El viejo enfermo deshumanizado}

Como mencionamos, las sociedades hipercognitivas que describe Post (2000) valoran el cerebro funcional como el órgano de la vida, también el razonamiento, la memoria y el aprendizaje, reconocidos como funciones intelectuales, al mismo tiempo que desvalorizan a los individuos que no demuestran tener una capacidad plena para utilizar estas funciones, entre ellos los enfermos de Alzheimer, las personas mayores y quienes sufren de un hándicap cognitivo y han perdido su "poder de" y su "poder sobre".

El enfermo de Alzheimer se define entonces por su carencia: no posee autocontrol, independencia,

1 El informe Warnock oficializa la tesis del "pre-embrión". Esta terminología se utiliza hoy en los comités de ética en investigación humana en Europa y Estados Unidos. 


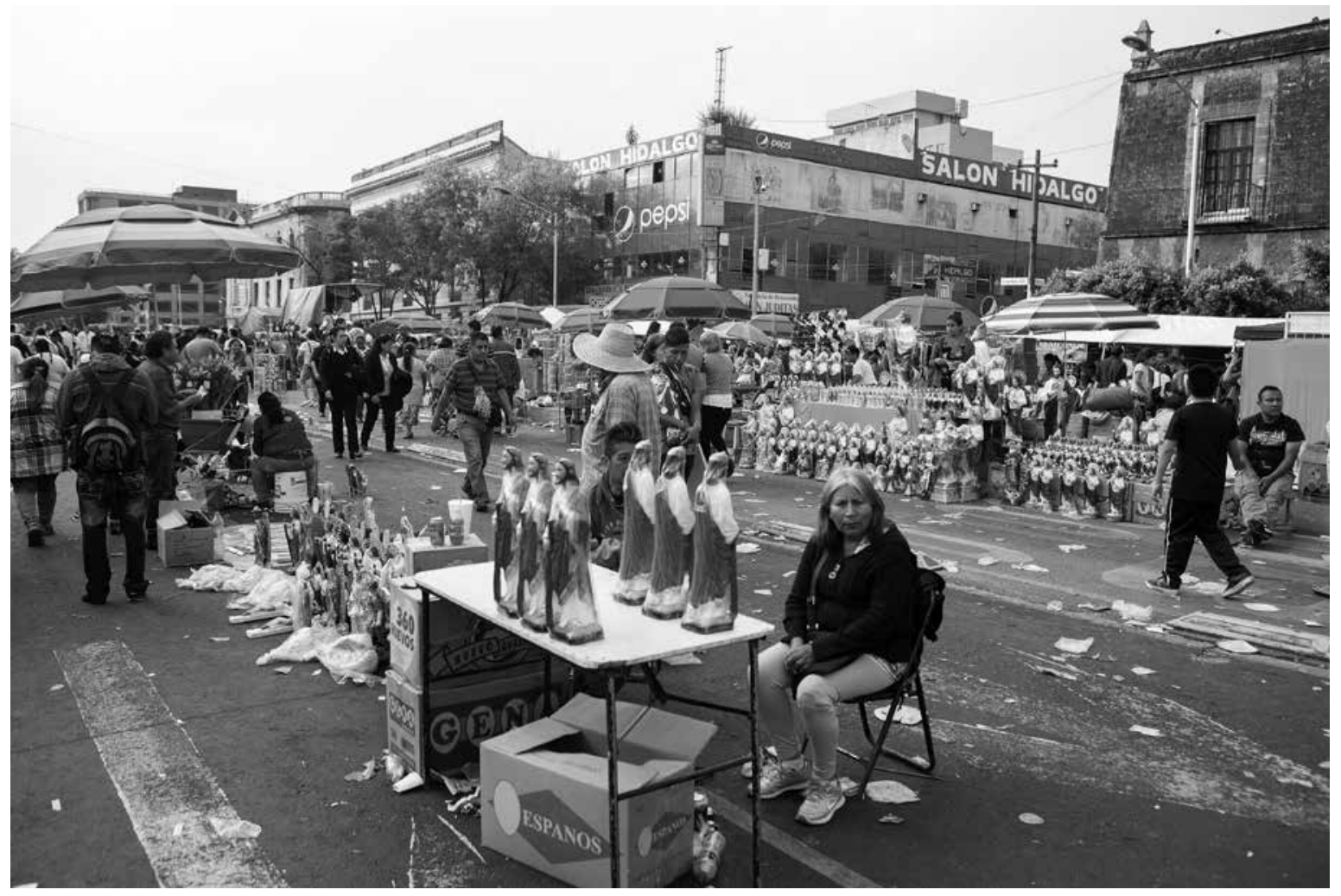

Prometeo Lucero - Comercio ambulante durante las celebraciones de san Judas Tadeo, Templo de San Hipólito, Ciudad de México, 28 de octubre de 2017.

productividad económica ni competencia cognitiva (Post, 2000: 162). El individuo desviado, en este caso el viejo enfermo, representado hoy por el enfermo de Alzheimer, es alejado y marginado. Su cuerpo se relega a las fronteras de la humanidad en la medida en que los demás lo perciben como "niño", "animal”, "vegetal” o "mineral" (Pierron, 2010: 104). Hoy, "el cuerpo viejo" es un "otro". Según Michel Foucault, "las representaciones humanistas tradicionales de las edades de la vida" han desaparecido (2001: 105). Así, el viejo se inserta en un imaginario colectivo que lo cosifica, lo naturaliza y lo asocia a la idea insostenible de dependencia y muerte.

Como plantea Pierron, si no podemos percibir al viejo enfermo como un ser humano entero es porque no reconocemos en él "los signos exteriores que permiten identificarlo como ser humano - su figura, su postura, su capacidad de actuar personajes sociales, etc.—” (2010: 102). Robert Bogdan y Steven J. Taylor (1989) mencionan cuatro modalidades con las cuales el no enfermo "reconoce al enfermo como humano": 1) cuando se le atribuye una forma de pensar; 2) cuando se percibe su individualidad; 3) cuando es posible verlo y ser visto por él, y 4) cuando se le puede atribuir un papel social (citado en Herskovits, 1995: 156). El enfermo de Alzheimer permanece como humano mientras los demás pueden percibirlo de esta forma. Cuando esto no ocurre, lo que se observa en la interacción con el enfermo es el fracaso de sus capacidades cognitivas y la degradación de sus funciones motrices. El viejo enfermo se vuelve irreconocible para los demás, en ocasiones también para sí mismo, y se 
inscribe en el cuadro de lo que Pierron denomina un "humanismo del hombre precario" (2010: 102).

El desarrollo de la enfermedad de Alzheimer amenaza con la disolución del yo. Incluso los afectados pueden referirse a sí mismos en tercera persona del singular: ese cuerpo, él, etc. Pero desde nuestro punto de vista, el problema central no es la subjetividad "en desorden" del enfermo, sino la "intersubjetividad" como fuente principal de "patogenicidad" (Herskovits, 1995: 147).

Foucault plantea que ya no se disfruta de la idea de envejecer. Nos "tropezamos con el problema de la muerte" sin concebir más la vejez como "un momento positivo, un momento de cumplimiento" (2001: 106). Al contrario de lo que acontecía con el viejo en las sociedades grecorromanas, el del siglo XXI no es "aquel que adquirió toda la experiencia posible, aquel que va a ser soberano de sí mismo y que se satisface enteramente de él mismo" (2001: 105). La persona que está envejeciendo se engloba de manera progresiva en las categorías anónimas del moribundo o del muerto viviente, en las cuales las singularidades y las historias de vida particulares desaparecen. De este modo, la fractura simbólica entre vivo y muerto se desplaza para otorgar nuevos sentidos a la dicotomía joven-viejo.

La medicalización de la vejez, por un lado, y luego su alzheimerización, modifican el estatus social y biológico del viejo enfermo. Como señalamos, las decisiones políticas y médicas de incorporar o excluir individuos de la vida social con base en la determinación, no sólo de su funcionamiento cerebral, sino también de su "buen funcionamiento", colocan al enfermo de Alzheimer en el umbral entre la humanidad y la no humanidad, la vida y la muerte. En este escrito pretendemos aproximarnos a la producción práctica de esta ambigüedad y sus tensiones desde un enfoque teórico-metodológico que, centrado en la vida cotidiana y las prácticas de cuidado, busca captar las experiencias de envejecer y vivir con la enfermedad de Alzheimer.

\section{Marco teórico y metodología}

Este trabajo se elabora en torno a los resultados de la tesis de maestría Être malade d'Alzheimer: une anthropographie de la présence (Cesanelli, 2013), dirigida por Albert Piette y codirigida por Emmanuel Grimmaud, defendida en Francia en 2013, en el Laboratoire d'ethnologie et de sociologie comparative de la Universidad de París X-Nanterre La Défense. La investigación consistió en el seguimiento etnográfico de seis mujeres diagnosticadas con Alzheimer en estadios distintos de la enfermedad, que vivían tanto en sus hogares como en instituciones especializadas, con el objetivo de describir y analizar su mundo cotidiano, también llamado "mundo de la vida cotidiana” por Alfred Schütz: "el mundo de nuestras experiencias de vidas comunes e inmediatas (frecuentemente contrastado con el mundo objetivo de las ciencias)" (citado en Recoder, 2011: 84). Ese mundo ordinario es subvertido o "deshecho" por la irrupción de la enfermedad, que cuestiona la "actitud natural" con la que las personas se desenvuelven, plantea nuevos problemas y exige la construcción de un nuevo orden de cosas, "una nueva cotidianidad significativa” (Schütz, 1979; Alves, Rabelo y Souza, 1999).

Para la construcción del marco teórico que permitiera analizar en detalle los datos recolectados durante un año de trabajo de campo, nos inspiramos en autores cuyas obras se inscriben en corrientes de pensamiento más familiares a la filosofía, que se revelaron fundamentales en nuestro caso para dar testimonio de la dimensión central del padecimiento y la atención a formas de sufrimiento social. Entre otros, Schütz (2008), Maurice Merleau-Ponty (1979), Paul Ricœur (1990; 1996) y Albert Piette (2011) nos introdujeron en las propuestas de la fenomenología y la hermenéutica. Con el propósito de hacer posible una antropología existencial que se enfocara en los detalles de las interacciones y experiencias de los individuos, nos acercamos a la 
etnometodología a partir de Erving Goffman (1968) y Harold Garfinkel (2007), para captar los procesos interpretativos por medio de los cuales aquellos se extrañan y se reapropian una y otra vez de sus acciones de la vida cotidiana y de las instancias que las componen. Como en el caso de Agnes, una mujer transexual descrita por Garfinkel en la década de 1960, quien debe, a cada instante, pensar sus gestos, sus posturas, sus maneras de dirigirse a los demás, en otras palabras, su manera de estar para ser reconocida como mujer por la sociedad (2007: 232).

A partir de esta perspectiva, nos acercamos a un grupo de mujeres diagnosticadas con la enfermedad de Alzheimer. Esto nos permitió verlas no sólo como enfermas que vivían un estado patológico degenerativo, sino como seres humanos "haciéndose", construyendo una nueva existencia y nuevos vínculos. Así, en la tesis se procuró captar las experiencias de estas mujeres. Definimos experiencia como "la forma original por la cual los sujetos concretos vivencian su mundo [...], el medio por el cual el mundo se ubica de cara al agente social y, como tal, está siempre localizado en el tiempo y en el espacio" (Alves, 2011: 22). De esta manera, apuntamos a describirlas en tanto sujetos, unas y múltiples, que devenían otras con su enfermedad en su vida cotidiana.

Con este fin se registró el detalle de las acciones e interacciones de Mina, quien a los 58 años de edad presentaba los primeros signos clínicos de la enfermedad y residía con su esposo en un departamento de Neuilly, en la provincia de París. Madame Gris, madame Rose, madame Blanc y madame Bleue, todas de alrededor de 70 años de edad en el momento de la investigación, se encontraban en un estadio intermedio de la enfermedad y residían en un geriátrico especializado de Issy-les-Moulineaux, también en la provincia de París. Constantina, de 88 años de edad, vivía el último estadio de la enfermedad en su casa, con su hijo y una enfermera, en un pueblo de la provincia de Viterbo, Italia.
Esta etnografía implicó establecer una relación intensa con estas mujeres y sus otros significativos, y facilitó construir los datos en el nivel microsocial con estrategias como entrevistas, observación y registro de las modalidades de presencia y de acción, y procesos de interacción de las mujeres entre ellas, con el personal de salud y sus familiares. Asimismo, se grabaron videos y se tomaron fotografías.

$\mathrm{Al}$ entrar en contacto con la residencia geriátrica, nos encontramos con una población integrada sobre todo por mujeres y muy heterogénea en edad, estado de salud y diagnóstico médico. Si bien el geriátrico se presentaba como una institución especializada en enfermos de Alzheimer, la mayoría de sus residentes nunca había recibido ese diagnóstico. Con la colaboración de la psicóloga de la institución, quien fue nuestra guía durante el trabajo de campo en el geriátrico, delimitamos un grupo de personas susceptibles de ser incluidas en el estudio. Los tres hombres residentes fueron descartados porque ninguno había recibido el diagnóstico. Las cuatro mujeres mencionadas, Gris, Rose, Blanc y Bleue, integraron nuestro universo de análisis porque todas habían sido diagnosticadas, tenían casi la misma edad, presentaban cuadros clínicos similares y compartían la mayoría de las actividades desarrolladas por la psicóloga del geriátrico.

Al comienzo, el trabajo de campo consistió en una participación sistemática, de agosto de 2012 a junio de 2013, en los talleres a los que acudían estas mujeres: el taller de tejido, el almuerzo terapéutico y el taller de juegos de mesa, organizados y coordinados por la psicóloga. Participar en estos espacios grupales y sus rutinas nos dio la oportunidad de interactuar con ellas y ganar su confianza. La familiaridad con las residentes nos dio acceso a momentos privilegiados con ellas, por separado, para dar un paseo por el jardín del geriátrico o para charlar en sus habitaciones.

Además de las numerosas conversaciones que tuvimos en la sala de reposo de los empleados, 
entrevistamos a la psicóloga en dos ocasiones, al principio y al concluir el trabajo de campo. No pudimos entrevistar a otros trabajadores de la institución, como enfermeras y asistentes de vida, ${ }^{2}$ pues no aceptaron participar en la investigación con el argumento de falta de tiempo. La psicóloga explicó: "tienen mucho trabajo, muchos pacientes y poco tiempo para atender. Quieren estar aquí el menor tiempo posible, es alienante. Terminan sus horas y se van. Muchas no vuelven. Es que saben que [los pacientes] se van a morir, es una cuestión de tiempo" (entrevista, 17 de abril de 2012). Apuntaba a la cuestión del desgaste asociado a las condiciones de trabajo y a la especificidad de las relaciones con los residentes hombres y mujeres.

En septiembre de 2012 conocimos a Mina, quien aceptó con entusiasmo colaborar con la investigación. Desde septiembre hasta junio de 2013 nos encontramos de una a tres veces por mes, según su disponibilidad; al comienzo, sólo en su casa, luego en parques, cafeterías o museos. La mayoría de las conversaciones que tuvimos en su casa se grabaron y tomamos fotos. Su esposo no quiso ser entrevistado, decía que los encuentros eran "una cosa suya [de Mina]".

En diciembre de 2013, ante la imposibilidad de acercarnos a una persona en fase terminal de la enfermedad de Alzheimer en París y gracias a un contacto familiar, decidimos trabajar con Constantina, quien vivía las últimas fases de la enfermedad en su casa en Italia. Entre diciembre y enero de 2013 nos trasladamos allí. Pudimos observar la vida cotidiana en su casa, hacer nueve filmaciones y cuatro entrevistas — tres a la cuidadora y una al hijo—, además de grabar cinco conversaciones, tres con la cuidadora, una con el hijo y otra con su esposa.

A partir del análisis de algunas de las situaciones registradas, pusimos en evidencia la importancia del tiempo en el desarrollo de las actividades cotidianas de estas mujeres y la importancia de los vínculos con otros, como se muestra a continuación.
El enfoque etnográfico permitió acceder a las experiencias de una población que actúa, se comunica y se relaciona, acciones no reconocidas ni registradas hasta ahora.

\section{Resultados}

\section{MODALIDADES DE PRESENCIA Y DE ACCIÓN}

Con el propósito de evitar lo que podríamos denominar el relativismo cultural referido al enfermo, según el cual la enfermedad aparece como una entidad específica que impide el funcionamiento "normal" del cuerpo y construye al individuo, lo idealiza y lo reduce a ciertas características propias de la enfermedad, se describieron y analizaron las acciones e interacciones de las mujeres en términos de modalidades de presencia/ausencia en la acción, en situaciones particulares, más allá de su condición de enfermas o su estado de salud definido clínicamente. Louis Ploton, psiquiatra especializado en la enfermedad de Alzheimer, sostiene que las acciones de los individuos, enfermos o no enfermos, implican al menos dos modalidades de presencia/ausencia: la mentalización y el descanso (2012: 22-25). La primera implicaría un intenso trabajo de atención, por lo tanto, de fuerte presencia en la acción efectuada y el mundo asociado a ella. La segunda, en cambio, supondría la ejecución casi automática de una actividad, sin necesidad de enfocar atención en ella, que en oposición a la otra, se asociaría a un cierto grado de ausencia.

Retomaremos esta distinción de modalidades para describir el desempeño de Mina en su vida cotidiana y cómo resuelve sola, o no, algunas situaciones problemáticas.

2 Personas con una formación paramédica, no enfermeras, que prodigaban cuidados a los residentes, como aseo y alimentación. 


\section{Extracto 1}

El 16 de octubre de 2012, a la hora de la merienda, Mina se levanta con entusiasmo de su sillón y se dirige hacia la cocina para hacer el té. Saca de un armario una escalera, explica que "es muy chiquita" y que no llega hasta arriba sin "un poco de ayuda". Se ríe, dispone la escalera frente a los estantes altos de la cocina y pone un pie sobre el primer escalón: “a mi marido no le gusta que me suba, hace siempre todo por mí. Pero no me ayudes, ¿dale?”.

Mina, con mano segura, abre los anaqueles, extrae una olla, una tetera de porcelana y una cajita de azúcar. Baja y pone la escalera en su lugar. Manos sobre su cadera, durante unos segundos mira con detenimiento la cocina antes de seguir buscando utensilios en los cajones y repisas bajos. Luego de dos minutos de búsqueda, Mina dispone en un ángulo de la mesa dos tazas, la tetera, la olla, el té, el azúcar, las cucharas y los fósforos, como para tener todo a la mano antes de empezar el proceso de preparación del té: "entonces, ¿qué tengo que hacer?”, dice. "Espera, eh, jespera! Porque yo tengo que pensar... Entonces, eh... Entonces vamos a tomar el té, y vamos a tomar el agua... Vamos a poner el agua. ¡Voy a poner el agua!”.

Mina, firme en sus gestos, toma la olla y le pasa con energía un paño limpio, abre el grifo del agua fría y la llena. De golpe, exclama: “ipero si tengo una tetera!”. Vacía la olla y llena la tetera antes de ponerla sobre la hornilla apagada. Se da vuelta y mira distraída unos segundos el cielo gris de octubre a través de la ventana. De repente, como si su mente "volviera a la realidad", mira la olla mojada en la pileta y la coloca vacía sobre la estufa. “¡Ah! ¡Pero qué estoy haciendo!”, dice molesta mientras la llena de agua otra vez y la deposita la hornilla, que finalmente prende.

En un instante, Mina se da vuelta y empieza a revolver las cosas en sus cajones: "es mi marido, siempre agarra las cosas... yo después... Pero espera... tengo, tengo... tengo otra cosa". Concentrada, se agacha para alcanzar el fondo de uno de los estantes y saca con satisfacción el azúcar. Sonriendo, abre el cajón de los cubiertos, saca dos cucharas y pone un poco de azúcar en cada taza.

Según Ploton (2012), cuando una persona decide tomar un té, empieza el trabajo de mentalización, por ejemplo, pensar dónde están las cosas, qué tipo de té elegir, la temperatura del agua, dónde está su taza preferida, etc. Una vez que la acción de "preparar el té" está en curso, la persona, que cuenta con la experiencia de "preparar el té" en su cocina, con sus utensilios, puede empezar a ausentarse mentalmente de sus gestos, pensar en otras cosas, hablar con quien la acompaña, escuchar música, enojarse, etc. Poco a poco, si no encuentra ninguna dificultad u obstáculo para el desarrollo de la actividad, como un corte de luz, que se caiga la azucarera, la falta de la taza preferida, entra en la fase de descanso, su atención disminuye y puede realizar la acción de manera casi automática.

Cuando Mina "prepara el té”, el proceso cognitivo descrito, que podría resumirse como la sucesión de 1) mentalización; 2) descanso, y 3) automatización, funciona de manera distinta, la secuencia de las acciones no sigue un orden convencional. Por ejemplo, Mina llena y vacía la olla varias veces, pone agua fría en la tetera, etc. El proceso de mentalizacióndescanso-automatización es inestable y discontinuo. Mina comienza mentalizando — piensa y prepara todo lo necesario sobre su mesa-, pero a medida que entra en la fase de descanso — se distrae, hablasus acciones se interrumpen, no se encadenan de manera convencional, como sería calentar el agua, poner el té en la tetera, vertir el agua caliente en la tetera. Incluso sus gestos se enredan cuando deja de pensar en lo que está haciendo. En cambio, vimos que "tiene que pensar", preguntarse "qué estoy haciendo" y volver a ordenar sus ideas, abandonar la fase de descanso una y otra vez. Mina fluctúa entre descanso y trabajo de mentalización. Va y viene 
entre presencia y ausencia, entre la olla y la tetera, el agua fría y la caliente, lo que tiene que hacer y lo que no logra hacer, lo que tiene que pensar y lo que automatiza. No obstante, con tiempo y paciencia suficientes, Mina logra ensamblar una serie de gestos y acciones para preparar y servir el té.

\section{EL TIEMPO DE EXISTIR}

En otra situación, es imposible que Mina alcance su objetivo sola. En un relato suyo, extracto 2, cuenta que le resulta imposible cumplir con ciertas actividades sin pedir ayuda.

\section{Extracto 2}

El 3 de noviembre de 2012, en un café de Place de la Concorde, Mina narra alegre una anécdota sobre el loro de su hermana:

Mi hermana me pidió cuidar a su bebé [un loro recién nacido]. Se tenía que ir de vacaciones y era muy pequeño, necesitaba alguien que lo cuidara. Pero es difícil, ¿sabes? Tengo dificultades para cuidarme a mí misma, entonces pedirme esto... jla verdad! Pero bueno, fui a buscarlo la semana pasada, por suerte mi hermana me había dejado todas las instrucciones escritas. Pero la verdad no me imaginaba que una cosa tan chiquita pudiera tener tantas necesidades. También... cómo se dice... ¡tan exigente! Es muy exigente. Si la leche no está en la temperatura correcta no come. Primero hay que calentar la comida en el microondas y después ponerla en una pequeña... ¿ ¿ómo se dice, el cosito ese? Después tengo que tomar... ¿Cómo se llama el cosito ese que aspira?... ¡La jeringa! Una pequeña jeringa de plástico. Pero me tengo que apurar, hacerlo rápido, rápido, pasados los 40 grados no come. ¡Tengo que empezar todo de vuelta! Y yo no puedo. La verdad que no puedo cuando hay que apurarse... Forget it! Es muy frustrante, ¿sabes? Muy frustrante... Entonces llamé a una amiga para que veniera a ayudarme, una amiga italiana, Léa. No, es muy frustrante. Pero comió y, no, al final no se murió.

Las dificultades que Mina encuentra al darle de comer al lorito no se relacionan de manera directa con la ejecución de la actividad, sino con sus exigencias temporales. La sucesión de acciones para calentar la leche en el microondas a 40 grados, ponerla en la jeringa y darla al loro antes de que se enfríe tiene que desarrollarse en un lapso muy breve, pues el pájaro sólo toma leche a esa temperatura. Sin embargo, como vimos, las secuencias de acciones de Mina son discontinuas, y su presencia, fluctuante. Esto último no le permite completar su objetivo y necesita la intervención de su amiga Léa para alimentar al lorito. Tomarse su tiempo es una condición ineludible para que Mina realice con éxito una actividad por sí misma.

\section{ALZHEIMER, UNA CUESTIÓN DE COLABORACIÓN}

La enfermedad de Alzheimer, en términos médicos un padecimiento cerebral degenerativo, puede entenderse al mismo tiempo como la reescritura de uno mismo que se desarrolla en la relación con el otro. Como Mina, madame Gris y Constantina construyen su experiencia de la vida cotidiana por medio de las relaciones que mantienen con personas específicas, la psicóloga de la institución para la primera y la enfermera para la segunda. Si bien el grado de avance de la enfermedad de Alzheimer influye sobre el grado de dependencia de estas mujeres, todas viven la relación con el otro como un elemento fundamental en la construcción de su nuevo ser. Para ilustrar este propósito, describiremos un momento de intercambio entre la psicóloga y madame 
Gris durante el taller de tejido y luego mostraremos el vínculo que une a Constantina y su enfermera Marie-Thérèse.

\section{Extracto 3}

Como todos los miércoles a las 11:00 horas, en el primer piso de la institución Les Neuf Muses, el Club trico'thé reúne a algunas residentes alrededor de una mesa llena de lanas coloridas. El 17 de abril de 2012, Victoria, la psicóloga, ayuda con paciencia y buen humor a las señoras Gris, Rose, Blanc, Jaune y Rouge en el desarrollo de sus tejidos. Madame Gris, de 73 años de edad, a diferencia de las otras participantes, parece reticente a empezar la actividad y mantiene firme contra su pecho su cartera y un libro de Mary Higgins Clark. Victoria se da cuenta de la situación, se acerca a ella, se pone en cuclillas y con una mano sobre su pierna le pregunta: “¿cómo está hoy, madame Gris? ¿Le parece unirse a nosotras para tejer?”. Sonriendo, la mujer parece relajarse, suelta las cosas que tenía en las manos y acepta la invitación. Victoria empieza su propio tejido y le indica a cada una los pasos a seguir. Pero madame Gris la interpela de nuevo: “¿yo qué tengo que hacer?”. Con un tono diáfano, Victoria le explica que está haciendo un escarpín que combina con el gorro que terminó la semana anterior. Para ilustrar sus palabras, saca la prenda de una bolsa y la muestra a las demás: “¡miren qué lindo trabajo que hizo madame Gris la semana pasada!”. Las otras participantes del taller y las enfermeras que pasan por ahí felicitan con afecto a madame Gris, que responde con una sonrisa tímida. Victoria se dirige a ella: "mire, ¡mire qué lindo quedó!”. Sorprendida, pregunta si de verdad es obra suya. "Sí, señora, le garantizo que fue todo trabajo suyo", exclama Victoria mientras se acerca y le enseña el gorro. "Es verdad que es lindo este gorrito", responde madame Gris, regocijada. "Sí, hermoso. Y ahora usted hará unos escarpines que combinen. ¡Mire!”.
Victoria presenta el trabajo que la mujer ha hecho hasta ese momento, tres líneas y 20 puntos derechos: "ahora, madame Gris, usted tiene que cerrar las ocho primeras con las ocho del final y después tiene que tejer 18 nudos más". Ella repite las indicaciones de Victoria, y decidida, empieza a tejer. Sus manos, que el tiempo ha deformado, emplean la lana y las agujas con destreza y velocidad. Tarareando, la precisión de sus gestos da testimonio de su experiencia. Luego de unos minutos, madame Gris interrumpe su trabajo y busca con la mirada la atención de Victoria: “¿ahora qué tengo que hacer?”. "Sí, ¿terminó de cerrar? ¿Qué tiene que hacer ahora?”. Deja pasar unos segundos, mira su trabajo y le confirma a la psicóloga que terminó de cerrar. Victoria le indica cómo seguir. Madame Gris retoma en silencio su trabajo con la misma agilidad y Victoria dirige su atención hacia madame Blanc.

La temporalidad extendida en la percepción de un individuo es lo que permite apreciar una sucesión de instantes como un todo continuo y homogéneo. Edmund Husserl, retomado por Jean Naudin, explica este fenómeno con una metáfora musical:

Cuando por ejemplo una melodía resuena, el sonido individual no desaparece del todo cuando se detiene el estímulo [...]. Cuando un sonido resuena, el precedente no desaparece sin dejar una huella, de lo contrario, seríamos incapaces de distinguir las relaciones entre los sonidos que se encadenan uno tras otro: tendríamos a cada instante un único sonido o, eventualmente, tendríamos, en el intervalo de tiempo, el tintineo de dos sonidos y una fase vacía entre ellos, pero jamás la representación de una melodía. Por otro lado, no se trata de hacer que se queden las representaciones de los sonidos en la conciencia. Si se quedaran, en efecto, sin modificaciones, tendríamos, en vez de una melodía, un acorde hecho de sonidos simultáneos, o algo más similar a una cacofonía (Naudin, 1997: 115). 


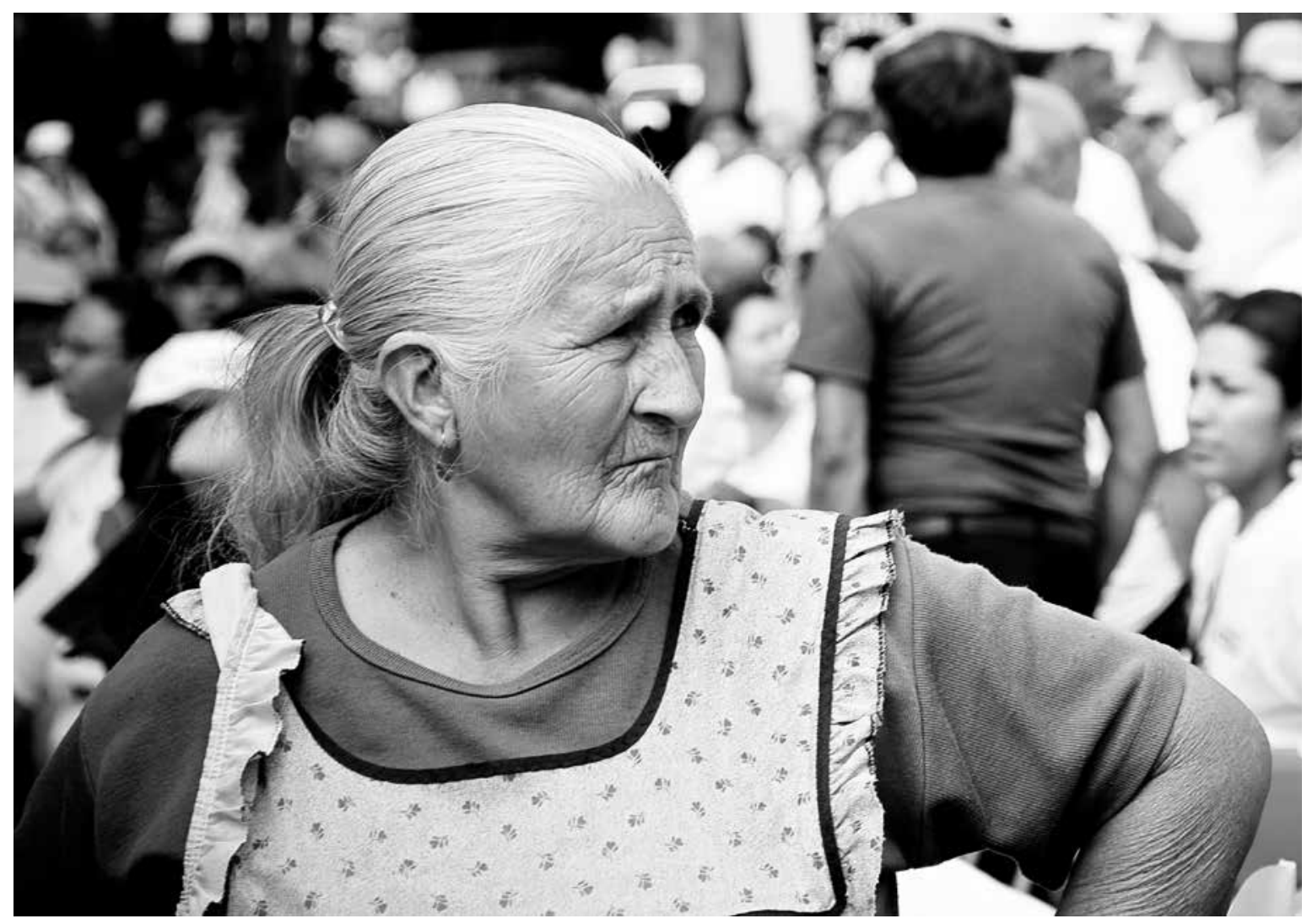

Rodrigo González • Colonia Tabacalera, Ciudad de México, abril de 2008.

Según este razonamiento, una melodía existe como tal sólo porque somos capaces de percibirla en su profundidad temporal. En otras palabras, podemos relacionar los sonidos pasados y presentes y esperar los futuros para que formen un todo.

El individuo que padece la enfermedad de Alzheimer pierde poco a poco esta temporalidad de la percepción que describe Husserl. La discontinuidad de sus acciones y la dificultad para unirlas hacen que el enfermo de Alzheimer empiece a necesitar en cierto punto la intervención de otro para darle homogeneidad y profundidad. Por ejemplo, cuando madame Gris teje, no logra encadenar sola la serie de puntos para armar su tejido, recurre a Victoria para saber lo "que tiene que hacer" después. Paso a paso, cada vez que pierde el hilo conductor de su trabajo, la psicóloga no sólo le explica de nuevo las etapas del proceso de creación del escarpín, también (re)contextualiza cada una y las inscribe, de alguna manera, en un continuum con un antes y un después. Juntas, madame Gris y Victoria, entre olvido y perseverancia, arman con lentitud las tramas del tiempo y el tejido.

\section{EL CUIDADO Y LAS MICROINTERACCIONES}

Las actividades de Constantina se resumen en pocos gestos. Está en una situación de dependencia total y parece vivir en un estado letárgico. MarieThérèse, su cuidadora desde hace tres años, hace todo por ella. Desde una mirada superficial y breve, 
Constantina podría caracterizarse como una persona que ha dejado de interactuar y decidir, de aceptar o negar los cuidados, de expresar sufrimiento o deseo. Parece que vive en un estado vegetativo, no habla, no se levanta de la cama, no se mueve. Esa descripción superficial coincidiría con las representaciones del viejo deshumanizado que vimos antes, que ha perdido su autonomía y sus funciones cognitivas. Pero la observación constante y atenta de algunos momentos importantes de las jornadas de Constantina, como las comidas o los aseos, reveló, en sus ritmos lentos y casi imperceptibles, las secuencias de acciones y las microestrategias de comunicación que utiliza para hacerse entender y manejar ciertas situaciones.

\section{Extracto 4}

Son las 9:15, la hora del desayuno se acerca. Gracias a una especie de grúa, concebida para personas mayores con movilidad reducida, Marie-Thérèse traslada a Constantina, limpia y vestida, de su cama al sillón acolchonado de tela verde, que la recibe todos los días a la misma hora. A su izquierda, hay una silla de madera antigua para un eventual invitado. A su derecha, en un elegante tocador quedaron algunos objetos que "antes" usaba siempre, un espejo de bronce, un cepillo de nácar, un portacollar, una cajita de maquillaje de plata y una botella de vidrio con perfume. En Radio-María se escuchan las oraciones de la mañana mientras Marie-Thérèse calza las pantuflas a Constantina antes de ir a la cocina a preparar su desayuno.

Cinco minutos más tarde, Marie-Thérèse vuelve y un rico olor invade la habitación. Tararea un canto religioso y lleva sobre una bandeja un tazón de leche chocolatada caliente y un paquete de galletas de manteca. Marie-Thérèse se sienta al lado de Constantina y apoya la bandeja sobre la mesita de luz. "¿Tenés hambre, Nonna? Vas a ser buena hoy y vas a comer todo, ¿sí?”. Para ambas, las comidas son un desafío de todos los días, ya lo saben. MarieThérèse rompe unas galletitas en la leche y tranquiliza a Constantina lo mejor que puede. Con voz afectuosa y caricias sobre su mano le da ánimo: "dale, vamos, vas a ver que está rico el desayuno y que va a estar todo bien. Va a pasar rápido. ¿Vamos?”.

Apenas la cuchara se acerca a su boca, Constantina aprieta los dientes. "Nonna, abrí la boca. Por favor. Muy bien”. La ingestión de comida parece desagradable para Constantina, casi dolorosa, gime, rechina los dientes y escupe. A cada cucharada, precede una negociación. Algunos días son más difíciles que otros, pero hoy Marie-Thérèse está contenta. Me dice: "casi comió todo y resistió poco". Le limpia la boca y la felicita: “¡brava Nonna! ¡Qué buena fuiste hoy! ¡Y comiste todo! Estoy contenta, muy contenta. Chocá los cinco". Despacio, Constantina responde a las solicitudes de su compañera de aventuras y le aprieta la mano de manera casi imperceptible.

Para terminar, Constantina tiene que hidratarse. Esa mañana tomará una mezcla de agua y jugos de naranja, mandarina y zanahoria. "Imposible darle agua, la escupe, no hay forma. Sería mejor que tome agua, seguro, por sus riñones y su estómago, pero no hay forma, la verdad". Marie-Thérèse le pasa un paño húmedo sobre la boca y la felicita de nuevo.

"La mayoría de las veces", dicen Franck Guihet y Antoine Hennion, "la auxiliar de vida está sola con sus propios recursos humanos, su sentido común y su empatía para adaptar su comportamiento, sus gestos y sus acciones" (2009: 119). El trabajo de cuidador es tedioso y cansador, requiere paciencia y una atención constante. Como lo dejó entender la psicóloga del geriátrico, en las instituciones de cuidado el tiempo requerido para atender las necesidades de cada individuo y establecer cierta empatía con él no está disponible. A este déficit de tiempo 
en las instituciones, que limita el vínculo con los enfermos, se agrega la precariedad de la condición del viejo enfermo, que es situado socialmente en el umbral entre humanidad y no humanidad, entre vida y muerte. El trabajo de cuidar a estos sujetos suele ser frustrante y agotador, y carece de la perspectiva de un desenlace favorable, dada la construcción de un ciclo degenerativo inevitable con el único horizonte de un tratamiento orientado hacia una muerte más o menos inminente.

Sin embargo, el caso de Constantina y MarieThérèse plantea algunas diferencias. Sin querer idealizar o romantizar su relación, tenemos que admitir que durante las entrevistas Marie-Thérèse en ningún momento expresó cansancio, frustración o agotamiento. Es importante aclarar que Constantina no siempre estuvo para indicar el camino a su cuidadora. En una de las entrevistas, Marie-Thérèse narró que al principio Constantina no comía con ella: "no abría la boca, durante días y días no quería comer, y ¡no comió! Pensaba que se me iba a morir. Yo le decía a su hijo, quizá piensa que la quiero envenenar, ¡no sé! [risas]. Pero un día empezó a comer. Comió y... un poco más cada día”.

A lo largo de los tres años que pasaron juntas, Marie-Thérèse aprendió poco a poco "el lenguaje de su abuelita", que "detestaba" que le limpiara el interior de la boca, un cuidado necesario que debía "negociar" y hacer de la manera más rápida posible. ${ }^{3}$ Sabía también que la sensación del guante tibio sobre su cara la aliviaba o que cuando "se niega categóricamente" a comer es porque le "duele la panza".

Cuando observamos los vínculos y los momentos de cooperación entre Marie-Thérèse y Constantina, una que practica el cuidado y la otra que lo acepta y guía con ruidos, microexpresiones y pequeños gestos, apreciamos el desarrollo de una relación sólida basada en el afecto y la cercanía, no en la interacción "performativa" a la que hicimos referencia más arriba. Constantina reconocía el gesto de Marie-Thérèse, lo traducía y respondía por medio de un esquema de comunicación construido durante una larga y particular experiencia emocional y sensible.

\section{Conclusión}

Si bien las ciencias sociales han hecho aportes importantes al conocimiento de los problemas relativos a la vejez y sus consecuencias políticas, económicas e institucionales, existen pocos estudios sobre los procesos de atención de las personas afectadas por Alzheimer, que se concentran en general en el acompañamiento institucionalizado. En esos trabajos resaltan las dificultades de los cuidadores, enfermeros y asistentes de vida al trabajar con esta población (Ancet, 2010). En nuestra investigación, la perspectiva etnográfica permitió ir más allá de la consideración de cuerpos y mentes de individuos enfermos y su deterioro inevitable para colocar la mirada sobre el proceso material, simbólico y afectivo del acompañamiento y los cuidados. En este marco, nos aproximamos a lo que implica para una persona transformarse a medida que la enfermedad avanza, y cómo, a lo largo de esta transformación, el otro que comparte la vida se vuelve siempre más íntimo y cercano. Cuida, apoya, guía, pero también otorga sentido y significado a interacciones cada vez menos evidentes, a esas performances no "dirigidas" pero que constituyen la reproducción y vida cotidiana de las personas afectadas.

Consideramos que el enfoque relacional aplicado, centrado en la experiencia de la enfermedad, en tanto realidad construida por procesos significativos compartidos de manera intersubjetiva

Marie-Thérèse cuenta que al principio Constantina la mordía, pero ahora que "saben" cómo hacerlo, no la muerde más. 
(Alves, 2006), puede aportar una mejor apreciación de la complejidad de la vida y los cuidados de las personas que viven con Alzheimer y colaborar a una mejor comprensión de los requerimientos y necesidades de atención en las etapas del proceso de la enfermedad. Al mismo tiempo, puede contribuir a cuestionar el modelo dominante de la "carencia" en el estudio de la vejez y el envejecimiento en el desarrollo del ciclo vital humano. D

\section{Bibliografía}

Adelman, Richard C., 1995, "The Alzheimerization of Aging", en The Gerontologist, vol. 35, núm. 4, pp. 526-532.

Alves, Paulo C., 2006, "A fenomenologia e as abordagens sistêmicas nos estudos sócio-antropológicos da doença: breve revisão crítica”, en Cadernos de Saúde Pública, vol. 22, núm. 8, pp. 1547-1554.

—_ 2011, "Presentación. Comprendiendo la clínica médica. Breve introducción teórico-metodológica”, en María Laura Recoder, Vivir con VIH-sida. Notas etnográficas sobre el mundo de la enfermedad y sus cuidados, Biblos, Buenos Aires, pp. 17-26.

Alves, Paulo C., Míriam Cristina Rabelo y lara Souza, 1999, "Introdução", en Míriam Cristina Rabelo, Paulo C. Alves y lara Souza (eds.), Experiência de doença e narrativa, Fiocruz, Río de Janeiro, pp. 11-39.

Ancet, Pierre, 2010, "Virilité, identité masculine et hándicap”, en Albert Ciccone (dir.), Handicap, identité sexuée et vie sexuelle, Éditions Érès, Toulouse, pp. 157-170

Ballenger, Jesse, 2003, "Beyond the Characteristic Plaques and Tangles: Mid-Twentieth Century U.S. Psychiatry and the Fightagainst Senility", en Peter J. Whitehouse, Konrad Maurer y Jesse Ballenger (eds.), Concepts of Alzheimer Disease: Biological, Clinical, and Cultural Perspectives, The Johns Hopkins University Press, Baltimore, pp. 83-103.

Bick, Katherine L., 1994, “The Early Story of Alzheimer Disease”, en Robert D. Terry, Robert Katzman y Katherine L. Bick (eds.), Alzheimer Disease, Raven Press, Nueva York, pp. 1-8.

Blanc, Guillaume Le, 2002, "Le conflit des médecins”, en Esprit, vol. 5, núm. 284, pp. 71-86.

Bogdan, Robert y Steven J. Taylor, 1989, "Relationships with Severely Disable People: The Social Construction of Humanness", en Social Problems, vol. 36, núm. 2, pp. 135-148.

Cesanelli, Violetta, 2013, Etre malade d'Alzheimer: une anthropographie de la présence, tesis de maestría, Laboratoire d'ethnologie et de sociologie comparative, Université Paris X-Nanterre, Nanterre.

Conrad, Peter y Arthur Schneider, 1992, Deviance and Medicalization. From Badness to Sickness, Temple University Press, Filadelfia.

Foucault, Michel, 2001, L'herméneutique du sujet. Cours au Collège de France (1981-1982), Seuil, París.

Garfinkel, Harold, 2007, Recherche en ethnométhodologie, Presses Universitaires de France, París.

Goffman, Erving, 1968, Asiles. Études sur la condition sociale des malades mentaux, Éditions de Minuit, París.

Guihet, Franck y Antoine Hennion, 2009, “Vivre avec Alzheimer, vivre avec un 'Alzheimer”, en Gérontologie et Société, vol. 32, núms. 128-129, pp. 117-128.

Herskovits, Elizabeth, 1995, "Struggling over Subjectivity: Debates about the 'Self' and Alzheimer's Disease", en Medical Anthropology Quarterly, vol. 9, núm. 2, pp. 146-164.

Katzman, Robert, 1976, “The Prevalence and Malignancy of Alzheimer's Disease: A Major Killer”, en Archive of Neurology, vol. 33, núm. 4, pp. 217-218.

Kraepelin, Emil, 1903, Psychiatrie: ein Lehrbuch für Studierende und Ärzte, J. A. Barth, Leipzig.

Lafontaine, Céline, 2010, “La vieillesse, une maladie mortelle”, en Revue Internationale de Soins Palliatifs, vol. 25, pp. 5-9.

Lock, Margaret, 2004, "Living Cadavers and the Calculation of Death", en Body and Society, vol. 10, núms. 2-3, pp. 135-152.

Merleau-Ponty, Maurice, 1979, Le visible et l'invisible, Gallimard, París.

Naudin, Jean, 1997, Phénoménologie et psychiatrie: les voix et la chose, Presses Universitaires du Mirail, Toulouse.

Ngatcha-Ribert, Laëtitia, 2012, Alzheimer: la construction sociale d'une maladie, Dunod, París.

Peatrik, Anne-Marie, 2003, "Jacques Dupâquier, ed., L'Espérance de vie sans incapacité. Faits et tendances, premières tentatives d'explication", en L’Homme, núms. 167-168, pp. 319-320. 
Pierron, Jean-Philippe, 2010, “Corps vieillissant, corps vil?”, en Pierre Ancet (dir.), Le corps vécu chez la personne âgée et la personne handicapée, Dunod, París, pp. 101-115.

Piette, Albert, 2011, Fondements à une anthropologie des hommes, Hermann Éditeurs, París.

Ploton, Louis, 2012, "Ce que nous enseignent les malades d'Alzheimer”, en Geneviève Arfeux-Vaucher y Louis Ploton (dirs.), Les démences au croisement des non-savoirs, Presses de l'École des hautes études en santé publique, Rennes, pp. 17-32.

Post, Stephen, 2000, The Moral Challenge of Alzheimer Disease: Ethical Issues from Diagnosis to Dying, The Johns Hopkins University Press, Baltimore.

Recoder, María Laura, 2011, "Experiencia de enfermedad y narrativa. Notas etnográficas sobre vivir con VIH/sida en una ciudad del Nordeste brasilero", en Papeles de Trabajo, núm. 21, pp. 80-98.

Ricœur, Paul, 1990, Soi-même comme un autre, Seuil (L’ordre philosophique), París.

_- 1996, Soi-même comme un autre, Seuil, París.

Roca, Alejandra, 2013, "Entre vivos y muertos", ponencia presentada en la IV Reunião de Antropologia da Ciência e da Tecnologia, Instituto de Filosofia e Ciências Humanas, Universidade Estadual de Campinas, 25 de septiembre, Campinas.

Schütz, Alfred, 1979, "Bases da fenomenologia”, en Alfred Schütz, Fenomenologia e Relaçoes Sociais, Zahar Editores, Río de Janeiro, pp. 53-76.

-, 2008, Le chercheur et le quotidien. Phénoménologie des sciences sociales, Méridiens Klincksieck, París.

Tavernier, Jean-Luc (dir.), 2016, Tableaux de l'économie française (TEF), Institut national de la statistique et des études économiques (Collection Insee Références Références Mesurer pour comprendre), París.

“The Warnock Committee”, 1984, en British Medical Journal, vol. 289, pp. 238-239.

\section{Entrevistas}

Psicóloga, residencia Les Neufs Muses, París, 17 de abril de 2012. 